\title{
Cryptococcemia in a Patient with COVID-19: A Case Report
}

\author{
Mohamad Khatib ${ }^{1}$, Amna Ahmed ${ }^{1}$, Said Shaat ${ }^{1}$, Ahmed soliman Mohamed ${ }^{1}$, and \\ Abdulqadir Nashwan ${ }^{1}$ \\ ${ }^{1}$ Hamad Medical Corporation
}

October 22, 2020

\begin{abstract}
Cryptococcus neoformans infection is a known cause of meningoencephalitis in immunocompromised patients. However, it is less frequent in immunocompetent individuals. Here we report a case of a 60 years old COVID-19 patient with a history of several co-morbidities who received tocilizumab and corticosteroids, which lead to Cryptococcemia.
\end{abstract}

\section{Key Clinical Message:}

The authors urge clinicians to balance the use of Immunosuppressant drugs and tocilizumab for COVID-19 patients to avoid the development of infections like Cryptococcemia, leading to death within 30 days.

\section{Background:}

Cryptococcus neoformans are basidiomycetous; encapsulated yeasts are classified into 4 serotypes according to their capsule. (1) the way of the infection is by inhalation of basidiospore form or small, poorly encapsulated yeasts. (2) Cryptococcus neoformans infection typically happens among immunocompromised patients and meningoencephalitis is the typical manifestation of cryptococcosis among HIV patients. $(3,4)$ in immunocompetent, the infection can be subclinical and a study on postmortem immunocompetent just showed small granulomatous disease in the lung without disseminating the infection. $(5,6)$ We describe a case of a patient with COVID-19 who developed Cryptococcemia while being treated by immunosuppressive treatment.

\section{Case Presentation}

A 60-year-old man with a history of hypertension, diabetes mellitus, and ischemic heart disease admitted to the intensive care unit (ICU) with confirmed COVID-19 infection required mechanical ventilation; on the same day of admission, he was pronned for many times due to low $\mathrm{PaO} 2 / \mathrm{FiO} 2(\mathrm{P} / \mathrm{F})$ Ratio. He received 3 doses of tocilizumab (400mg/once), (600mg/once) and $(600 \mathrm{mg} /$ once) respectively due to possible cytokine storm; he received several doses of methylprednisolone as part of the COVID-19 management and hydrocortisone.

Few days after receiving tocilizumab, his bronchial alveolar lavage was positive for Candida glabrate; he was started on anidulafungin $200 \mathrm{mg}$ q $24 \mathrm{hr}$ for 7 days. The patient was sedated and intubated for 22 days, so a tracheostomy was done. He developed acute kidney injury (AKI) after 28 days of ICU admission hence hemodialysis started.

Despite that the patient was on antibiotics, his inflammatory marker is still high. Thus, full septic workups were done, and the patient was having candidemia (Candida parapsilosis ) after one month of the admission. For which he was started on anidulafungin $200 \mathrm{mg}$ q $24 \mathrm{hr}$ for more than 7 days. Many episodes of gastrointestinal (GI) tract bleeding complicated his course manifested as melena and per rectal bleeding, but all the episodes were managed conservatively by the gastroenterology team. The candidemia persists after 2 
weeks of treatment and there was an increase in C-reactive protein (CRP) level despite that the patient was under antibiotic and antifungal cover. Hence, the ID team recommended giving antifungal (anidulafungin $100 \mathrm{mg}$ ) for a total of 14 days to involve an ophthalmologist to rule out fungal ophthalmitis. Still, it was not applicable and to do an echocardiogram to rule out vegetation and it was ruled out by trans-thoracic echocardiogram.

While the patient was on anidulafungin, his blood culture became positive for Cryptococcus neoformans after 18 days of receiving anidulafungin for previous candida infection, for which he was started on amphotericin (300mg, q24hr) and flucytosine (500mg q12hr). And there was a recommendation from the ID team to do trans esophageal echo and lumbar puncture to rule out vegetation and brain involvement, respectively, but both tests were not done due to poor prognosis and risk of bleeding because the patient had thrombocytopenia (platelet 21, RR=150- $400 \times 10^{9} / \mathrm{L}$ ). The Cryptococcemia persist despite the management and he developed sepsis and die within 10 days of the Cryptococcemia.

\section{Discussion}

Disseminated Cryptococcus neoformans infection is a serious infection that can occur in immunocompromised patients. (7) During the outbreak of COVID-19 disease, there were many studies about the overwhelming immune response to COVID-19 infection through the activation of a large number of $\mathrm{T}$ lymphocytes and mononuclear macrophages, producing cytokines like interleukin-6 (IL-6), which bind to the receptor and cause cytokine storm and severe inflammation in the lung and other tissues. Tocilizumab, as an anti-human IL-6 receptor monoclonal antibody and immunosuppressant drugs, was recommended by several studies for alleviating the inflammatory response. (8)

Jean and colleagues have noted in their study of 52 patients withCryptococcus neoformans isolated from blood culture, the primary three predisposing for Cryptococcemia are Acquired immunodeficiency syndrome, immunosuppressive therapy and decompensated liver cirrhosis. (9) And in our case, the Cryptococcus neoformans was isolated from the blood culture. And according to Passerini M et al that Cryptococcus neoformans can affect different orangs as meningoencephalitis, which is the most common, followed by pulmonary and cutaneous disease. (10)

According to Jean et al that patients with positive blood culture for Cryptococcus neoformans developed sepsis by $81 \%$, including those developed septic shock, the lumbar puncture (LP) was positive in $61.5 \%$ of patients with positive blood culture for Cryptococcus neoformans and $84 \%$ of those with LP positive showed meningeal involvement, in our case LP was recommended but not done because the patient was critically sick and unstable. Also it was noted by Jean et al that the fatality within 30 days of Cryptococcemia was $37 \%$. (9) Also, in our case, the patient died within 10 days of Cryptococcemia. Therefore, it is essential to suspect Cryptococcus neoformansinfection in an immunocompromised patient and early management, keeping in mind the high morbidity associated with it. Consequently, it is crucial to avoid misdiagnosis and mismanagement, keeping in mind the high morbidity.

\section{Conclusion}

This case highlights the importance of early suspicion ofCryptococcus neoformans infection and other opportunistic infections in immunocompromised patients, putting in mind that patients with Cryptococcaemia have a high risk of mortality within 30 days, which warrants the use of corticosteroid and immunomodulatory drugs in a critically ill patient with COVID-19. In the current scenario, the use of immunosuppressive therapy should be justified and to be alert for opportunistic infection like Cryptococcus neoformans infection, which can lead to sepsis and mortality.

\section{Abbreviations \\ COVID-19: Coronavirus disease}

ICU: Intensive care unit

DM: Diabetes Mellites 
HTN: Hypertension

IHD: Ischemic Heart Disease

IL-6: Interleukin 6

HIV: Human Immunodeficiency Virus

LP: Lumbar Puncture

Declarations

Ethics approval and consent to participate

The article describes a case report. Therefore, no additional permission from our Ethics Committee was required.

\section{Consent for publication}

The consent for publication was obtained.

Availability of data and material

All data generated or analyzed during this study are included in this published article.

\section{Competing interests}

The authors declare that they have no competing interests.

\section{Funding}

This study was not funded.

\section{Authors' contributions}

MYK, AAA, SBS, ASM, AJN: Data Collection, Literature Search, Manuscript Preparation

All authors read and approved the final manuscript

\section{Acknowledgments}

Open Access funding provided by the Qatar National Library.

\section{Reference:}

1. Franzot SP, Salkin IF, Casadevall A. Cryptococcus neoformans var. grubii: separate varietal status for Cryptococcus neoformans serotype A isolates. Journal of clinical microbiology. 1999;37(3):838-40.

2. Velagapudi R, Hsueh Y-P, Geunes-Boyer S, Wright JR, Heitman J. Spores as infectious propagules of Cryptococcus neoformans. Infection and immunity. 2009;77(10):4345-55.

3. Cox G, Perfect J. Cryptococcus neoformans var. neoformans and gattii and Trichosporon species. Topley and Wilson's Microbiology and Microbial Infections, 9th ed London: Arnold Press p. 1997:461-84.

4. Lee SC, Dickson DW, Casadevall A. Pathology of cryptococcal meningoencephalitis: analysis of 27 patients with pathogenetic implications. Human pathology. 1996;27(8):839-47.

5. Baker RD. The primary pulmonary lymph node complex of cryptococcosis. American journal of clinical pathology. 1976;65(1):83-92.

6. Baker RD, Haugen RK. Tissue changes and tissue diagnosis in cryptococcosis. A study of 26 cases. American journal of clinical pathology. 1955;25(1).

7. Bamba S, Lortholary O, Sawadogo A, Millogo A, Guiguemde RT, Bretagne S. Decreasing incidence of cryptococcal meningitis in West Africa in the era of highly active antiretroviral therapy. Aids. 2012;26(8):1039-41. 
8. Xu X, Han M, Li T, Sun W, Wang D, Fu B, et al. Effective treatment of severe COVID-19 patients with tocilizumab. Proceedings of the National Academy of Sciences. 2020;117(20):10970-5.

9. Jean SS, Fang CT, Shau WY, Chen YC, Chang SC, Hsueh PR, et al. Cryptococcaemia: clinical features and prognostic factors. Qjm. 2002;95(8):511-8.

10. Passerini M, Terzi R, Piscaglia M, Passerini S, Piconi S. Disseminated Cryptococcosis in a Patient With Metastatic Prostate Cancer Who Died in the Coronavirus Disease 2019 (COVID-19) Outbreak. Cureus. $2020 ; 12(5)$. 